Polymer Journal, Vol. 7, No. 4, pp 498-506 (1975)

\title{
Synthesis of Reversible Oxygen Complex of Polymeric Hemochrome
}

\author{
Eishun Tsuchida and Kenji Honda \\ Department of Polymer Chemistry, Waseda University, Tokyo 160, Japan.
}

(Received January 6, 1975)

\begin{abstract}
Iron-protoporphyrin IX was bound to poly(vinylpyridine) and poly(vinylimidazole) at the axial site of the iron atom. The equilibrium constant for the complexation of heme with the polymeric ligand is $10^{2}$ times as large as that of the monomeric analogue. Such a polymeric hemochrome can take up molecular oxygen $\left(\mathrm{O}_{2}\right)$ more rapidly than does the corresponding monomeric hemochrome. The degree of oxygen saturation of the polymer complex is appreciably higher than that of the monomer complex.

The rate constant of oxygenation in water $\left(k_{\mathrm{a}} \simeq 10 \mathrm{sec}^{-1}\right)$ is much larger than that in an organic solvent $\left(k_{\mathrm{a}} \simeq 10^{-4} \mathrm{sec}^{-1}\right)$. The rate constant $\left(k_{\mathrm{a}}\right)$ at the adsorption step is appreciably affected by the conformational change of the polymer ligand; $k_{\mathrm{a}}$ is enhanced by about 10 times under the optimal conditions.

On the other hand, the rate constant $\left(k_{\mathrm{d}}\right)$ at the decomposition step of the oxygen complex is drastically affected by the environment around an active site. Upon adding an appropriate agent such as a salt, polyelectrolyte, or surface-active agent, $k_{\mathrm{d}}$ decreased drastically with increasing concentration of additives, so that an oxygen complex remained stable even in an aqueous solution.

KEY WORDS Iron--Protoporphyrin IX / Poly(vinylpyridine) / Poly(vinylimidazole) / Oxygenation / Discharge / Oxygen Complex / Environmental Effect /
\end{abstract}

When an effective oxygen carrier is designed, the following three factors should be considered: (a) stability of oxygen complex, (b) reversibility of oxygenation, and (c) efficiency of reversible adsorption-desorption of $\mathrm{O}_{2}$. In this paper, we will be concerned mainly about problem (a). An oxygen complex has been believed to be unstable in solutions, especially in an aqueous solution; $;^{1,2}$ perhaps this results from the rapid irreversible oxidation which is caused by the dissociation of the ion-pair of the oxygen complex. Therefore, in order to retard the irreversible oxidation, it is desirable that an active site of hemochrome be included in the hydrophobic cavity which is formed by a polymeric axial ligand of heme. ${ }^{3}$ We have succeeded in the synthesis of a stable oxygen complex in solutions of polymeric hemochrome by retarding the irreversible oxidation of the oxygen complex, which had been considered to be unavoidable for a monomeric hemochrome, by taking advantage of the hydrophobic enviromental effect of the polymer chain.

\section{EXPERIMENTAL}

\section{Synthesis and Purification of Reagents}

Isolation of Chlorohemin from Blood. Blood $(25 \mathrm{ml})$ and $0.9-\% \mathrm{NaCl}$ aqueous solution $(20 \mathrm{ml})$ were carefully mixed thoroughly, and then centrifuged at $3000 \mathrm{rpm}$ for $5 \mathrm{~min}$. The red precipitate was mixed with $20 \mathrm{~m} l$ of $0.9-\% \mathrm{NaCl}$ aqueous solution and centrifuged again. This procedure was repeated five times. The resulting red precipitate was mixed with $50 \mathrm{ml}$ of $0.9-\% \mathrm{NaCl}$ aqueous solution. This suspension was poured into a heated solution $\left(>93^{\circ} \mathrm{C}\right)$ of $300 \mathrm{ml}$ of acetic acid and $0.12 \mathrm{~g}$ of $\mathrm{NaCl}$ in a boiling water bath. The pouring must be performed quickly and with vigorous stirring. After the completion of pouring, stirring was continued for $10 \mathrm{~min}$ and the mixture was permitted to stand for about $12 \mathrm{hr}$ at room temperature. The dark-brown needle crystals that precipitated were filtered off, washed with 1-\% hydrochloric acid several times, and dried in vacuo.

$$
\text { Synthesis of Poly(4-vinylpyridine) (PVP) and }
$$




\section{Synthesis of Reversible Oxygen Complex of Polymeric Hemochrome}

Poly(N-vinyl-2-methylimidazole) (PVMI). PVP and PVMI were synthesized by the radical polymerization of 4-vinylpyridine and $\mathrm{N}$-vinyl-2methylimidazole, respectively, under the following conditions: [monomer] $=2.0 \mathrm{~mol} / l,[\mathrm{AIBN}]=$ $0.05 \mathrm{~mol} / \mathrm{l}$, in methanol at $70^{\circ} \mathrm{C}$ for $6 \mathrm{hr}$. The resulting polymers were poured into a large excess of ethyl ether; further purification was carried out by repeating this process. The monomers used were previously treated and then purified by distillation according to the usual method.

Synthesis of Partially Quaternized Poly(vinylpyridine) $(Q P V P)$. Poly(4-vinylpyridine) $(8.42 \mathrm{~g})$ was dissolved in $750 \mathrm{ml}$ of methanol and benzyl chloride $(10.1 \mathrm{~g})$ dissolved in $50 \mathrm{~m} l$ of methanol. The polymer solution was charged into a fournecked round-bottom flask fitted with a dropping funnel; the benzyl chloride solution was added from the dropping funnel and stirring was continued at $60^{\circ} \mathrm{C}$ for $12 \mathrm{hr}$. The mixture was then condensed to about $50 \mathrm{~m} l$ and poured into $1000 \mathrm{ml}$ of ethyl ether. The quaternized poly(vinylpyridine) obtained was dissolved in methanol and poured into ethyl ether once more and dried in vacuo. The degree of quaternization was determined to be $23.1 \%$ by Volhard's method.

Purification of Other Reagents Used. Pyridine, dimethylformamide (DMF), and methanol $(\mathrm{MeOH})$ were purified by distillation before being used in the spectral measurements. Imidazole was recrystallized from benzene. Sodium hydrosulfite (special grade commercial reagent) was used as a reductant without purification.

\section{Reduction and Oxygenation of Heme}

Chlorohemin was reduced by $\mathrm{Na}_{2} \mathrm{~S}_{2} \mathrm{O}_{4}$ under the following conditions: [chlorohemin] $=1.0 \times$ $10^{-5} \mathrm{~mol} / l ;\left[\mathrm{Na}_{2} \mathrm{~S}_{2} \mathrm{O}_{4}\right]=1.0 \times 10^{-3} \mathrm{~mol} / l ;[\mathrm{QPVP}]=$ $1.0 \times 10^{-2}$ pyridine-unit $\mathrm{mol} / l$; in $\mathrm{DMF} / \mathrm{H}_{2} \mathrm{O}(1: 9)$ at room temperature under $\mathrm{N}_{2}$ atmosphere. In order to complete the reduction of hemin by $\mathrm{Na}_{2} \mathrm{~S}_{2} \mathrm{O}_{4}$, the reaction vessel must be completely filled with $\mathrm{N}_{2}$. Reduction proceeded rapidly under such conditions as those mentioned above and the absorption band at $397 \mathrm{~nm}$, assignable to the oxidized pyridine-hemichrome, shifted to the band at $422 \mathrm{~nm}$ characteristic of the reduced pyridine-hemochrome. When $\mathrm{Na}_{2} \mathrm{~S}_{2} \mathrm{O}_{4}$ was added in the equimolar concentration to a mixture of $\mathrm{DMF}-\mathrm{H}_{2} \mathrm{O}(1: 9)$ dissolving chlorohemin, the characteristic absorption band due to the reduced pyridine-hemochrome did not appear in the Soret region and the absorbance at $397 \mathrm{~nm}$ progressively decreased with time. But in DMF containing 3 vol $\%$ of water the corresponding absorption band due to the reduced-type hemochrome slowly appeared at $416 \mathrm{~nm}$ and then the absorbance continuously decayed. It may thus be preferable to add $\mathrm{Na}_{2} \mathrm{~S}_{2} \mathrm{O}_{4}$ in $10^{2}$ times as large as the molar concentration of the hemin solution.

After a 10-\% aqueous DMF solution of the reduced QPVP - heme complex was mixed with oxygen-saturated water of the equivalent portion, the spectral change with time in the visible region was followed in an atmosphere of oxygen. The absorbance at $422 \mathrm{~nm}$ of the reduced hemochrome decreases gradually with increasing absorbance at $406 \mathrm{~nm}$ through the isosbestic point (see Figure 1). Since this new absorption band at $406 \mathrm{~nm}$ can be shifted back reversibly to the absorption

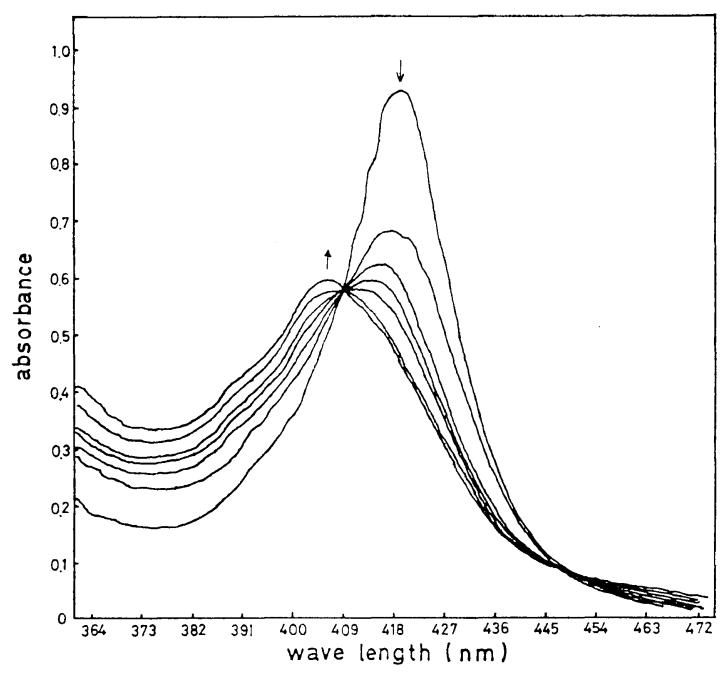

Figure 1. Spectral changes in the Soret region on exposure to oxygen for the QPVP-heme complex in $\mathrm{DMF}-\mathrm{H}_{2} \mathrm{O}(1: 9)$.

band at $422 \mathrm{~nm}$ by a nitrogen stream or degassing in vacuo, the absorption band at $406 \mathrm{~nm}$ should be assigned to the oxygenated hemochrome which coordinates a molecular oxygen to the 6th coordination position of heme-iron.

The rate constant $\left(k_{\mathrm{a}}\right)$ of oxygenation of heme was determined from the first-order plots of 
increase with time in absorbance at $406 \mathrm{~nm}$ under the condition of pseudo first-order reaction: i.e., that the concentration of oxygen dissolved in the solution $\left(10^{-3}-10^{-2} \mathrm{~mol} / l\right)$ is in large excess to the initial concentration of heme $\left(1.0 \times 10^{-5} \mathrm{~mol} / l\right)$. The rate constant $\left(k_{\mathrm{d}}\right)$ of irreversible oxidation of oxygenated heme was also determined from the first-order plots of decrease with time in absorbance at $406 \mathrm{~nm}$. The half-life period of the absorption band at $406 \mathrm{~nm}$ was used as a parameter of stability of the oxygen complex. The spectral measurements were carried out by a spectrophotometer (Shimadzu MPS-50L) and the rapid reactions were followed by a rapid scanning spectrophotometric analyzer (Union High-Sens, Spectrophotometer SM 401).

Polarographic Measurement of Redox Potential of Polymeric Hemichrome

The half-wave potential of reduction of hemin was measured by a Yanaco P-8 Type Polarograph (Yanagimoto Co., Ltd.), the cathode of which is a dropping mercury electrode and the anode of which is a mercury pool. The conditions of measurement were as follows: [Hemin] $=1.0 \times$ $10^{-5} \mathrm{~mol} / l,[\mathrm{QPVP}]=1.3 \times 10^{-3} \mathrm{~mol} / l$, in $\mathrm{H}_{2} \mathrm{O}-$ DMF $(9: 1)$.

\section{RESULTS AND DISCUSSION}

\section{Structures of Some Polymer-Heme Complexes}

In order to determine the coordination structures of hemichrome and hemochrome, the coordination number $(\tilde{n})$ of axial ligands, which is the ratio of axial ligand to heme, and an equilibrium constant $(K)$ were measured according to the Miller's method. ${ }^{4}$ Judging from the results shown in Table I, four planar coordination sites are occupied by porphyrin nitrogens and the axial 5 th site by a nitrogen atom of pyridine or imidazole. The 6th coordination site of the pyridine-hemichrome $\left(\mathrm{Fe}^{3+}\right)$ is occupied by a chloro or hydroxy ion in most cases. However, most of the coordination number of axial ligands shown in Table I show values intermediate between 1.0 and 2.0 , so that a bidentate pyridinehemichrome, whose axial sites are both occupied

Table I. Equilibrium constant and coordination number of axial ligands in the complexation of hemin and heme with pyridine and imidazole derivatives ${ }^{2}$

\begin{tabular}{|c|c|c|c|c|c|}
\hline \multirow{2}{*}{ Axial base } & \multicolumn{2}{|c|}{$K(l / \mathrm{mol})$} & \multicolumn{2}{|c|}{$\bar{n}$} & \multirow{2}{*}{ Solvent } \\
\hline & $\mathrm{Fe}(\mathrm{III})$ & $\mathrm{Fe}(\mathrm{II})$ & $\mathrm{Fe}(\mathrm{III})$ & $\mathrm{Fe}(\mathbf{I I})$ & \\
\hline Pyridine & 6.04 & $5.13 \times 10^{1}$ & 1.02 & 1.11 & \multirow{2}{*}{$\mathrm{DMF}-\mathrm{MeOH}(7: 3)$} \\
\hline PVP & $3.75 \times 10^{2}$ & $1.14 \times 10^{3}$ & 0.95 & $1.32\}$ & \\
\hline Imidazole & 9.52 & $4.55 \times 10^{1}$ & 1.14 & 1.20 & \multirow{2}{*}{$\mathrm{DMF}-\mathrm{MeOH}(1: 3)$} \\
\hline PVMI & $1.78 \times 10^{2}$ & $2.55 \times 10^{3}$ & 1.07 & $1.15\}$ & \\
\hline QPVP & $2.60 \times 10^{2}$ & $2.77 \times 10^{4}$ & 1.30 & 1.25 & $\mathrm{DMF}-\mathrm{H}_{2} \mathrm{O}(1: 9)$ \\
\hline
\end{tabular}

a [hemin], $1.0 \times 10^{-5} \mathrm{~mol} / l ;\left[\mathrm{Na}_{2} \mathrm{~S}_{2} \mathrm{O}_{4}\right.$ ], $1.0 \times 10^{-3} \mathrm{~mol} / l$, [axial base], $1.0 \times 10^{-3} \mathrm{~mol} / l$; at $25^{\circ} \mathrm{C}$; QPVP $\left(P_{n}=\right.$ $49, \mathrm{Q} \%=23.1$, quaternized by benzyl chloride).

Table II. Positions of the Soret bands of various heme complexes

\begin{tabular}{|c|c|c|c|c|c|}
\hline Solvent & Base added & Additives $^{\mathbf{a}}$ & $\begin{array}{c}\text { Oxidized type, } \\
\mathrm{nm}\end{array}$ & $\underset{\mathrm{nm}}{\text { Reduced type, }}$ & $\underset{\mathrm{nm}}{\text { Oxygenated type, }}$ \\
\hline DMF & - & - & 404 & 416 & - \\
\hline Pyridine & - & - & 422 & 382 & 390 \\
\hline \multirow{3}{*}{ DMF } & Pyridine (PVP) & - & 424 & 418 & 402 \\
\hline & Imidazole (PVMI) & - & 410 & 418 & 402 \\
\hline & QPVP & $\mathrm{NaCl}$ & 397 & 422 & 406 \\
\hline \multirow[t]{2}{*}{$\mathrm{H}_{2} \mathrm{O}$} & " & PMAA & 397 & 422 & 406 \\
\hline & Pyridine & NaLS & 406 & 422 & 408 \\
\hline
\end{tabular}

a $\left[\mathrm{NaCl}\right.$ ], about $0.35 \mathrm{~mol} / l ;[\mathrm{PMAA}]$, about $6 \times 10^{-4} \mathrm{~mol} / l ;[\mathrm{NaLS}]$, about $5 \times 10^{-2} \mathrm{~mol} / l$. 
by pyridine molecules, may be slightly formed in the mixture. The 6 th coordination site of the pyridine-hemochrome $\left(\mathrm{Fe}^{2+}\right)$ may be vacant or weakly occupied by a solvent molecule such as DMF or water in most cases. The structure of a ferrohemochrome is expected to be the "sitting-atop structure,,$^{5,6}$ in which the central iron atom is not situated in the plane of porphyrin but out of this plane. Moreover, the polymer-heme complexes are much more stable than the monomeric analogues in solutions.

Hemin was reduced in a dimethylformamide (DMF) solution or in an aqueous solution by $\mathrm{Na}_{2} \mathrm{~S}_{2} \mathrm{O}_{4}$ in a nitrogen atmosphere. After completion of reduction, aromatic amines such as pyridine, imidazole, and their polymeric derivatives were added to the reduced heme solution and molecular oxygen was brought into contact with the ferrohemochrome by bubbling oxygen gas. The positions of the Soret bands assignable to the oxidized-type, the reduced-type, and the oxygenated-type heme complexes are represented in Table II. The absorption bands which appeared at $402-408 \mathrm{~nm}$ on exposure to oxygen were assigned to the oxygenated hemochrome on the basis of the result shown in Figure 2.

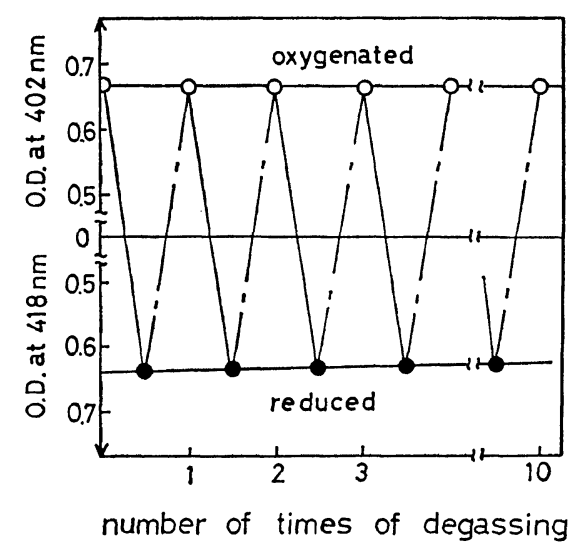

Figure 2. Reversible exchange between the absorption bands at 418 and $402 \mathrm{~nm}$ for the PVP-heme complex in DMF containing 3 vol $\%$ of $\mathrm{H}_{2} \mathrm{O}$.

That is, when the poly(vinylpyridine)-heme complex was alternately exposed to oxygen and degassed in a nitrogen stream, the new absorption band at $402 \mathrm{~nm}$ was reversibly exchanged for the absorption band at $418 \mathrm{~nm}$ due to the reduced-type hemochrome; each absorbance remained almost constant during the repeated procedures of bubbling and degassing oxygen.

Elementary Processes of the Reaction of Heme with Molecular Oxygen

Baldwin $^{7}$ and Collman ${ }^{8}$ have independently suggested that the irreversible oxidation of a ferrous complex proceeds as represented by eq 1 .

$$
\begin{aligned}
& \mathrm{Fe}(\mathrm{II})+\mathrm{O}_{2} \stackrel{\mathrm{a}}{\rightleftarrows} \mathrm{Fe}(\mathrm{II})-\mathrm{O}_{2} \\
& \underset{\mathrm{Fe}(\mathrm{II})}{\rightleftarrows} \mathrm{Fe}(\mathrm{II}) \mathrm{O}-\mathrm{O}-\mathrm{Fe}(\mathrm{II}) \longrightarrow \mathrm{Fe}(\mathrm{III})
\end{aligned}
$$

That is, the oxidation may be caused by the dimerization of a ferrous complex which produces an unstable peroxo complex. Therefore, in order to retard the dimerization and to make the reaction (a) of $\mathrm{Fe}(\mathrm{II})$ with $\mathrm{O}_{2}$ reversible, the ligand of the metal complex should be bulky. According to this reasoning, they have synthesized some individual model compounds which can take up oxygen reversibly in solutions at low temperature. But these compounds decompose at room temperature in contact with $\mathrm{O}_{2}$.

The present authors have succeeded in the synthesis of a stable oxygen complex of polymeric hemochrome which can take up $\mathrm{O}_{2}$ reversibly and effectively at room temperature. Equation 2 represents the elementary processes in the oxygenation. The first step is the adsorption process, in which a molecular oxygen is taken up by a ferrous ion. The second step

$$
\begin{aligned}
\mathrm{O}^{/ / O} & \mathrm{O}^{/ \mathrm{O}^{-}} \\
\mathrm{Fe}(\mathrm{II})+\mathrm{O}_{2} & \stackrel{k_{\mathrm{a}}}{\rightleftarrows}\left[\mathrm{Fe}(\mathrm{II}) \longleftrightarrow \mathrm{Fe}^{+}(\mathrm{III})\right] \\
\stackrel{k_{\mathrm{d}}}{\longrightarrow} & \mathrm{Fe}^{+}(\mathrm{III})+\mathrm{O}_{2}{ }^{-}
\end{aligned}
$$

is the discharge process in which an oxygen complex dissociates into two ion species from the dative structure. The electronic configuration of the oxygen complex has been studied extensively as a monomeric chelate, ${ }^{9-11}$ and the conclusion reports that two types of structures (the non-bonding and the dative structures) exist in the resonance form, as shown in eq 2 .

In a polar solvent, the dissociation of the ion pair is fast, so that the irreversible oxidation proceeds rapidly, especially in water. On the other hand, in a nonpolar solvent, the dissociation of the ion pair may be extremely retarded. 
Table III. Rate constants and activation parameters of oxygenation of pyridine - and imidazole-hemochromes ${ }^{a}$

\begin{tabular}{cccccc}
\hline Axial base & Solvent & $k_{\mathrm{a}}, \mathrm{sec}^{-1}$ & $E_{\mathrm{a}}, \mathrm{kcal} / \mathrm{mol}$ & $\Delta H^{\neq}, \mathrm{kcal} / \mathrm{mol}$ & $\Delta S^{\neq}$, e.u. \\
\hline Py & DMF-MeOH $(9: 1)$ & $0.99 \times 10^{-4}$ & 11.0 & 10.4 & -43.6 \\
PVP & $\prime \prime$ & $1.86 \times 10^{-4}$ & 26.9 & 26.4 & +8.85 \\
Im & $\prime \prime$ & $4.78 \times 10^{-4}$ & 10.1 & 9.44 & -43.4 \\
PVMI & $\prime \prime$ & $7.76 \times 10^{-4}$ & 19.2 & 18.6 & -13.2 \\
QPVP & $\mathrm{H}_{2} \mathrm{O}-$ DMF $(9: 1)$ & $1.07 \times 10^{\circ}$ & 23.0 & 22.4 & +13.0 \\
\hline
\end{tabular}

a at $40^{\circ} \mathrm{C}$.

If a polymeric ligand is used to include an active site of heme in the hydrophobic environment, the resulting oxygen complex is expected to be stable even in an aqueous solution. The kinetics of each elementary step in oxygenation have been studied in order to elucidate the conformational and environmental effects of the polymer ligand on the reversible oxygenation.

Adsorption of Oxygen by Polymeric Hemochromes

The oxygenation rates and their activation parameters for hemes bound to the synthetic polymers such as poly(4-vinylpyridine) (PVP), poly $(N$-vinyl-5-methylimidazole) (PVMI), and partially quaternized PVP (QPVP) were compared with those of the monomeric model compounds. As Table III shows, the oxygenation rates of the polymeric hemochromes are appreciably larger than those of the monomeric derivatives in systems of both pyridine and imidazole. Judging from the fact that the activation entropy of the polymeric system is larger than that of the monomeric system, the enhancement of reactivity of the polymeric hemochromes may be due to the thermal fluctuation of the polymer chain and the resulting increase in the frequency of collision between heme and $\mathrm{O}_{2}$.

The oxygen capacities were measured by a Warburg apparatus; the results are shown in Figure 3. The polymer complexes take up $\mathrm{O}_{2}$ much more than do the monomer complexes and the initial rates of the polymer complexes were also larger than those of the monomer complexes. Thus, it was found from the results of spectrometry and volumetric analysis that the activity of oxygenation of the polymeric hemochromes is generally higher than that of the corresponding monomeric hemochromes.

In order to examine the conformational effect

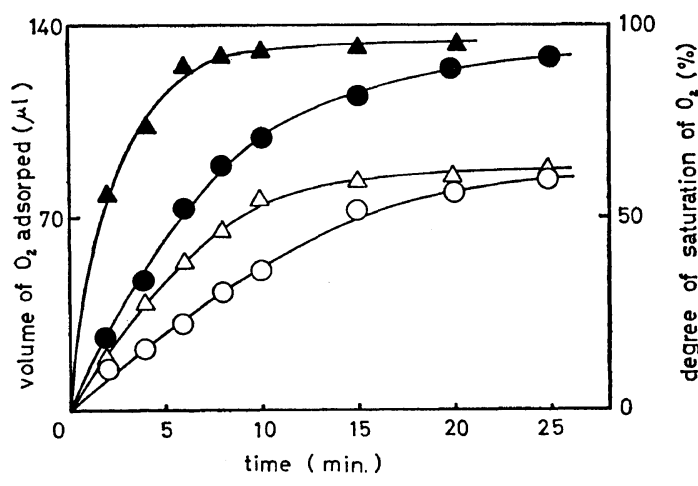

Figure 3. Oxygen up-take by various hemochromes: [hemin], $5.62 \times 10^{-3} \mathrm{~mol} / l$; [axial base], $5.62 \times 10^{-1}$ $\mathrm{mol} / l ;\left[\mathrm{Na}_{2} \mathrm{~S}_{2} \mathrm{O}_{4}\right], 4.50 \times 10^{-1} \mathrm{~mol} / l$; in DMF-MeOH (9:1); trace of $\mathrm{H}_{2} \mathrm{O}$ added; at $30^{\circ} \mathrm{C}$; $\bigcirc$, pyridine; ○, PVP; $\triangle$, imidazole; $\Delta$, PVMI.

of a polymeric ligand upon the activity of oxygenation of heme bound to a synthetic polymer, the relationship between the oxygenation rates and the composition of a mixed solvent has been studied for the PVP heme complex and the PVMI heme complex. As Figures $4 \mathrm{a}$ and $\mathrm{b}$ show, the reduced viscosities of PVP and PVMI increased by increasing the methanol fraction in volume of a mixed solvent, and at the same time, the equilibrium constants in the complexation of hemichrome decreased with the methanol fraction. From the steric hindrance, the expansion of polymeric ligand may be favorable for taking up $\mathrm{O}_{2}$ by heme bound to a polymeric ligand. However, this is unfavorable for oxygenation of heme from the other point, since the concentration of an active hemochrome preferentially participating in oxygen up-take decreases with the expassion of the polymer chain, as shown in Figures $4 \mathrm{a}$ and $\mathrm{b}$. These competitive factors operate simultaneously in real systems. Thus, 
Synthesis of Reversible Oxygen Complex of Polymeric Hemochrome

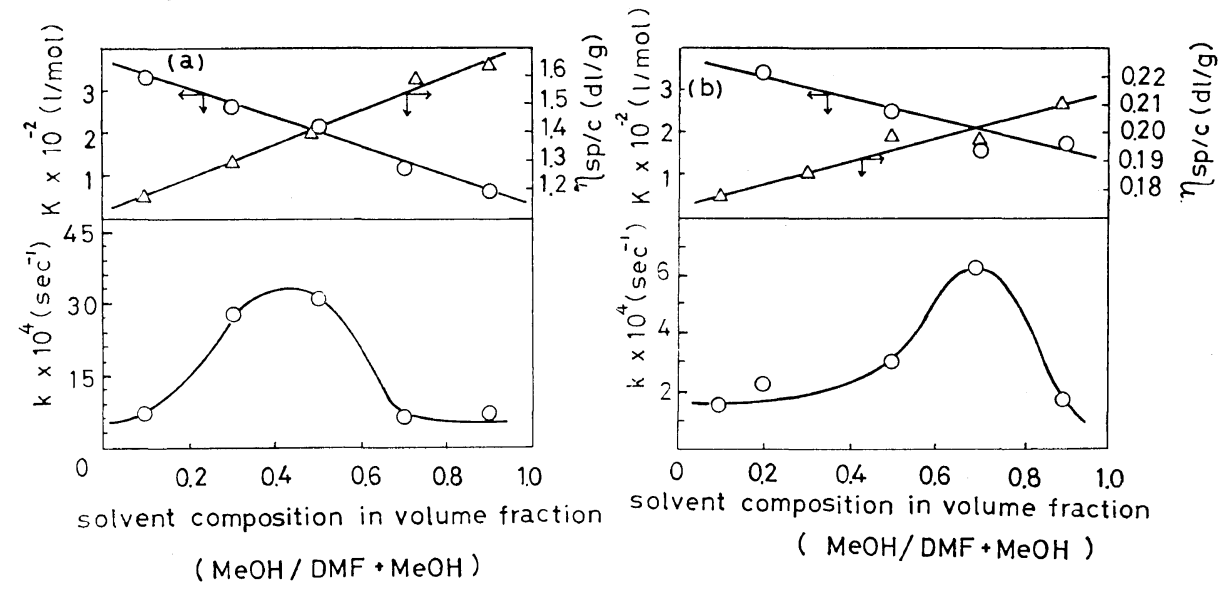

Figure 4. Conformational dependence of oxygenation rate of polymeric heme complex, (a) PVPheme system, (b) PVMI--heme system: [hemin], $1.0 \times 10^{-5} \mathrm{~mol} / l ;[\mathrm{PVP}], 1.0 \times 10^{-2} \mathrm{~mol} / l$; [PVMI], $1.0 \times 10^{-2} \mathrm{~mol} / l ;\left[\mathrm{Na}_{2} \mathrm{~S}_{2} \mathrm{O}_{4}\right], 8.0 \times 10^{-4} \mathrm{~mol} / l$.

Table IV. Solvent effect on dissociation of ion pair of oxygen complex

\begin{tabular}{|c|c|c|c|}
\hline Solvent & $\begin{array}{c}\text { Axial } \\
\text { base }\end{array}$ & Additives & $k_{\mathrm{d}}, \sec ^{-1}$ \\
\hline \multirow{2}{*}{ DMF } & $\mathrm{Py}$ & - & $2.72 \times 10^{-4}$ \\
\hline & PVP & & $3.83 \times 10^{-4}$ \\
\hline \multirow{2}{*}{$\mathrm{DMF}-\mathrm{Bz}(4: 1)$} & Py & & $1.04 \times 10^{-4}$ \\
\hline & PVP & & $1.78 \times 10^{-4}$ \\
\hline \multirow{3}{*}{$\mathrm{H}_{2} \mathrm{O}-\mathrm{DMF}(9: 1)$} & QPVP & $\mathrm{NaCl}$ & $73.7 \times 10^{-4}$ \\
\hline & " & PMAA & $0.23 \times 10^{-4}$ \\
\hline & Py & NaLS & $21.2 \times 10^{-4}$ \\
\hline
\end{tabular}

the bell-shape responses shown in these figures were observed. The oxygenation rate of the polymeric hemochromes could be enhanced about 10 times by the conformational change of the polymer chain.

\section{Irreversible Oxidation of Oxygen Complex}

Table IV shows that the rate constant of $k_{\mathrm{d}}$ at the discharge process (eq 2) in DMF is smaller than in water, and that $k_{\mathrm{d}}$ decreases by adding benzene to DMF. This result indicates that the rate constant of $k_{\mathrm{d}}$ is greatly affected by the polarity of the solvent used. In particular, in water the discharge of the ion pair of the oxygen complex occurs rapidly. Consequently, a stable oxygen complex has not been obtained in water. We tried to stabilize the oxygen complex of heme in an aqueous solution by using polymeric ligands and some additives.

We first discuss the reaction system of $\mathrm{NaCl}$.
When $\mathrm{NaCl}$ was added to an aqueous solution containing QPVP-heme complex, a polymer chain of QPVP became progressively more compact as the concentration of $\mathrm{NaCl}$ was raised, as Figure 5a shows. The rate constant of $k_{\mathrm{d}}$ at the discharge of the ion pair decreased gradually with increasing concentration of $\mathrm{NaCl}$. The decrease in $k_{\mathrm{d}}$ is predictable: the reason for this is that the active site of heme may be situated in the hydrophobic environment formed by shrinkage of the polymer chain of QPVP.

Similar results were obtained in the system of poly(methacrylic acid) (PMAA) (Figure 5b). When PMAA was added to an aqueous solution dissolving the QPVP - heme complex, the reduced viscosity of the mixed solution decreased progressively with the increasing concentration of PMAA; the minimum point appeared at some concentration of PMAA where the molar ratio of quaternized pyridinium group of QPVP to carboxylic group of PMAA was near 1.0. The reduced viscosity of the polymer solution became gradually larger above this concentration. This result reveals that QPVP can bind PMAA mainly by electrostatic interaction and that the resulting interpolymer complex has almost an equimolar composition of each component. Also in this case, the rate constant of $k_{\mathrm{d}}$ decreased drastically with the decreasing viscosity of solution. That is, until the molar ratio of the quaternized pyridinium group of QPVP to the carboxylic group of PMAA became near 1.0, $k_{\mathrm{d}}$ decreased 

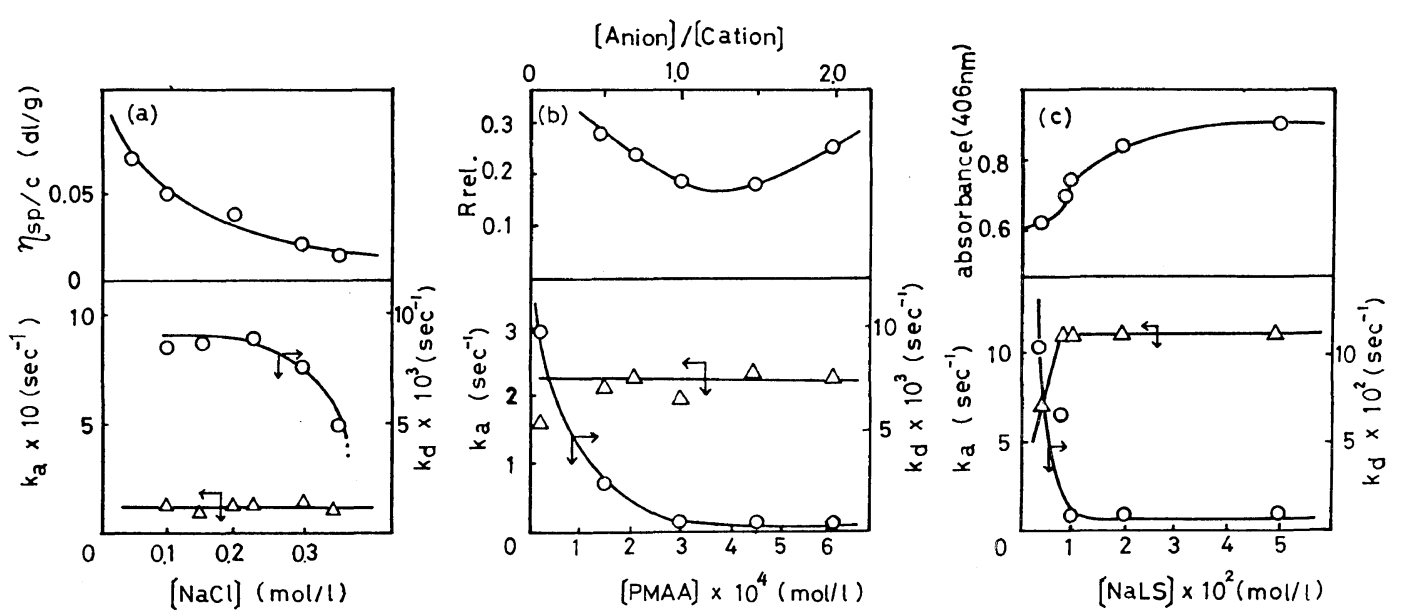

Figure. 5 Hydrophobic environmental effect on the kinetics of elementary processes of oxygenation, (a) QPVP-NaCl system, (b) QPVP-PMAA system, and (c) pyridine-NaLS system: [hemin], 1.0 $\times$ $10^{-5} \mathrm{~mol} / l$; [QPVP], $1.0 \times 10^{-2} \mathrm{~mol} / l\left(\mathrm{Q} \%=23.1, \overline{P_{n}}=49\right) ; \quad\left[\mathrm{Na}_{2} \mathrm{~S}_{2} \mathrm{O}_{4}\right], 1.0 \times 10^{-3} \mathrm{~mol} / l$, in $\mathrm{H}_{2} \mathrm{O}-\mathrm{DMF}$ $(9: 1)$; at $25^{\circ} \mathrm{C}$. (b): $R_{\mathrm{rel}}=\eta_{\mathrm{sp}} / C(\mathrm{QPVP}+\mathrm{PMAA}) /\left[\eta_{\mathrm{sp}} / C(\mathrm{QPVP})+\eta_{\mathrm{sp}} / C(\mathrm{PMAA})\right]$.

progressively with the increasing concentration of PMAA. And $k_{\mathrm{d}}$ became approximately constant above this concentration. This phenomenon may also result from an increase in hydrophobicity around heme by binding the QPVP-heme complex to PMAA.

In addition, micellaneous systems in which a surface-active agent binds the monomeric pyridine-hemochrome into the hydrophobic cavity formed by micell was also studied in comparison with the polymeric systems. Figure $5 \mathrm{c}$ shows that $k_{\mathrm{d}}$ dropped quickly in the vicinity of the critical micell concentration of sodium lauryl sulfate (NaLS), i.e., $8.0 \times 10^{-3} \mathrm{~mol} / l$.
Thus, it could be also found that inclusion of heme into the hydrophobic cavity of micell results in retardation of the discharge of an ion pair of the oxygen complex.

\section{Conformation Dependence of Redox Potential of}

Central Metal Ion of Polymeric Hemichrome

We have studied the relationship between the reactivity and the redox potential of metal complex. In order to relate the kinetical behavior of $k_{\mathrm{a}}$ and $k_{\mathrm{d}}$ to the redox potential of QPVPhemichrome, the half-wave potentials of polymeric hemichrome were measured by polarography. Figures $6 \mathrm{a}-\mathrm{c}$ show the results obtained when the additives were $\mathrm{NaCl}$, PMAA, and $\mathrm{NaLS}$.
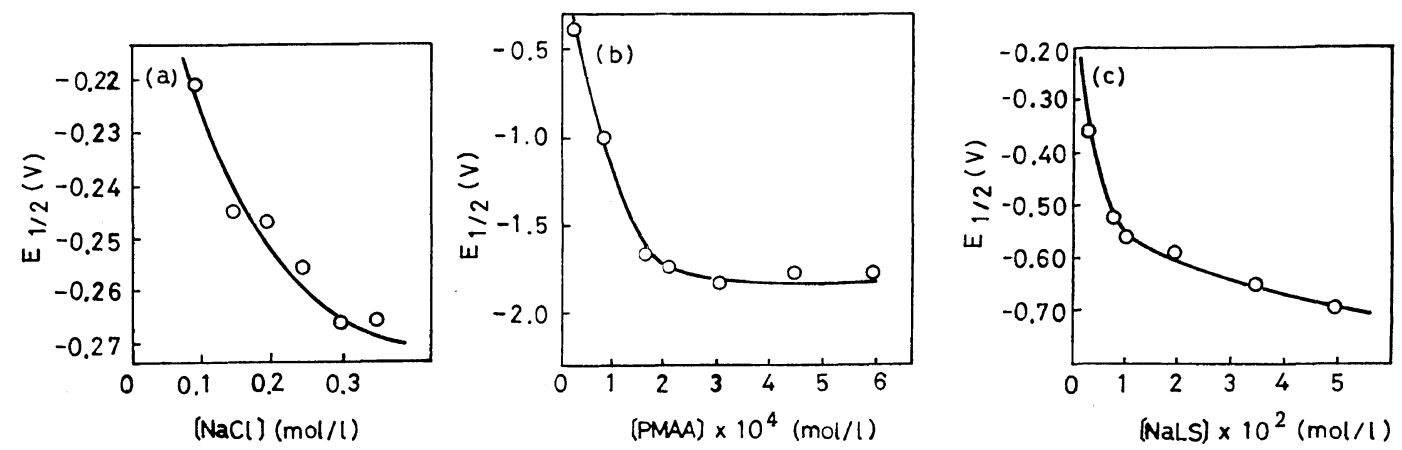

Figure 6. Relationship between half-wave potential of pyridine-hemichrome and concentration of additives, (a) QPVP - NaCl system, (b) QPVP_PMAA system, and (c) pyridine-NaLS system: [hemin], $1.0 \times 10^{-5} \mathrm{~mol} / l ;$;QPVP], $1.0 \times 10^{-2} \mathrm{~mol} / l$; in $\mathrm{H}_{2} \mathrm{O}-\mathrm{DMF}(9: 1)$. 
All results show that the larger the hydrophobicity around heme becomes by adding salt, polyeletrolyte, or other materials in high concentration, the lower the redox potential of heme becomes. These results are consistent with the kinetic features of the discharge process in oxygenation. Judging from this result, the redox potential of the central metal ion must be low in order to retard the irreversible oxidation of heme. However, if the redox potential is too low, the affinity of metal for $\mathrm{O}_{2}$ becomes small. Therefore, it is desirable for the redox potential to remain within a certain range. The optimal potential for reversible oxygenation of heme in an aqueous solution may be about $-1.0 \pm 0.5 \mathrm{~V}$ vs. $\mathrm{Hg}$ pool.

\section{Stability of Oxygen Complex of Polymeric Hemo- chrome}

Studies on the oxygenation of monomeric hemochromes have concluded that an oxygen complex is very unstable in water at room temperature because of the rapid irreversible oxidation. Recently, some investigators ${ }^{7,8,12,13}$ succeeded in the stabilization of oxygen complex of heme at low temperature below $-50^{\circ} \mathrm{C}$ in solutions, as already mentioned above. Even in these cases, it was observed that the oxygen complex decomposed into an unknown compound at room temperature. We have found that the characteristic absorption band of the oxygenated hemochrome at $402 \mathrm{~nm}$ decayed immediately when the monomeric hemochrome was brought into contact with $\mathrm{O}_{2}$ at room temperature. But if the polymeric ligand of QPVP was used with monomeric salt or polyelectrolyte, the absorption band at $402 \mathrm{~nm}$ persisted much longer than in the monomeric complex, as shown in Table $\mathrm{V}$. When a surface-active agent was used, the oxygen complex was stabilized as much as in the case

Table V. Stability of oxygen complex of pyridine-hemochromes in an aqueous solution

\begin{tabular}{cc}
\hline Additives & Retention time, sec \\
\hline Py & $1 \pm 0.1$ \\
QPVP $+\mathrm{NaCl}$ & $30 \pm 2$ \\
Py $+\mathrm{NaLS}$ & $30 \pm 5$ \\
QPVP + PMAA & $3000 \pm 60$ \\
\hline
\end{tabular}

of a monomeric salt. Thus, we can conclude that the stabilization of the oxygen complex of polymeric hemochrome is caused by retardation of the separation of the ion pair of the resulting oxygen complex, owing to the hydrophobic microenvironment of the polymer chain around heme.

However, it cannot be completely denied yet that the dimerization between the ferrous ions of heme through the peroxo bridge as shown in eq 1 takes place also under the experimental conditions presented in this paper. If such is the case, it is more probable that the decomposition of the resultant peroxo complex is retarded, since the flexibility of polymer chains is decreased due to the cross-linkage through the peroxo bridge. The Wang's model of the synthetic hemochrome ${ }^{3}$ is negative according to the supplementary experiments performed by Hatano. ${ }^{14}$ It is therefore necessary to examine the mechanism of the oxidation of heme by $\mathrm{O}_{2}$ more clearly. We are now trying to detect such an intermediate species, especially in the decomposition process of the oxygen complex.

\section{REFERENCES}

1. A. H. Corwin and Z. Reyes, J. Amer. Chem. Soc., 78, 2437 (1956); A. H. Corwin and S. D. Bruck, ibid., 80, 4736 (1958).

2. H. Kobayashi and Y. Yanagawa, Bull. Chem. Soc. Japan, 45, 450 (1972).

3. J. H. Wang, J. Amer. Chem. Soc., 80, 3168 (1958); Accounts Chem. Res., 3, 3168 (1970).

4. J. R. Miller and G. D. Dorough, J. Amer. Chem. Soc., 74, 3977 (1952).

5. J. L. Hoard, M. J. Hamor, T. A. Hamor, and W. S. Caughey, ibid., 87, 2312 (1965).

6. D. M. Collins, R. Countryman, and J. L. Hoard, ibid., 94, 2066 (1972).

7. J. F. Baldwin and J. Huff, ibid., 95, 5757 (1973); Chem. Eng. News, 51 (35), 11 (1973).

8. J. P. Collman, R. Gangne, T. R. Halbert, J. Marchon, and C. A. Reed, J. Amer. Chem. Soc., 95, 7868 (1973); Chem. Eng. News, 52 (3), 20 (1974).

9. G. N. Schrauzer and L. P. Lee, J. Amer. Chem. Soc., 92, 1551 (1970).

10. J. Peisach, W. E. Blumberg, B. A. Wittenberg, and J. B. Wittenberg, J. Biol. Chem., 243, 1871 (1968).

11. A. L. Crunbliss and F. Basolo, J. Amer. Chem. Soc., 92, 55 (1970). 
H. Tsuchida and K. HondA

12. C. K. Chang and T. G. Traylor, Proc. Nat. Acad. Sci. U. S., 70, 2647 (1973); J. Amer. Chem. Soc., 95, 5810, 8475, 8477 (1973).

13. T. Ouhadi, A. J. Hubert, Ph. Teyssie, and E.
G. Derouane, ibid., 95, 6481 (1973).

14. M. Hatano, Chemistry and Chemical Industry (The Chemical Society of Japan), 18, 926 (1965). 